\title{
Projectivity and unification in the varieties of locally finite monadic $M V$-algebras
}

\author{
Antonio Di Nola ${ }^{1}$, Revaz Grigolia'; , and Giacomo Lenzi ${ }^{3}$ \\ 1 University of Salerno, Salerno,Italy \\ adinola@unisa.it \\ 2 Tbilisi State University, Tbilisi, Georgia \\ revaz.grigolia359@gmail.com \\ ${ }^{3}$ University of Salerno, Salerno, Italy \\ gilenzi@unisa.it
}

\begin{abstract}
A description of finitely generated free monadic $M V$-algebras and a characterization of projective monadic $M V$-algebras in locally finite varieties is given. It is shown that unification type of locally finite varieties is unitary.
\end{abstract}

\section{Introduction}

Monadic $M V$-algebras (monadic Chang algebras by Rutledge's terminology) were introduced and studied by Rutledge in [5] as an algebraic model for the predicate calculus $q L$ of Lukasiewicz infinite-valued logic, in which only a single individual variable occurs. Rutledge followed P.R. Halmos' study of monadic Boolean algebras. In view of the incompleteness of the predicate calculus the result of Rutledge in [5, showing the completeness of the monadic predicate calculus, has been a great interest. Adapting for the propositional case the axiomatization of monadic $M V$-algebras given by Rutledge in [5, we can define modal Lukasiewicz propositional calculus $M L P C$ as a logic which contains Lukasiewicz propositional calculus $L u k$, the formulas as the axioms schemes: $\alpha \rightarrow \exists \alpha, \exists(\alpha \vee \beta) \equiv \exists \alpha \vee \exists \beta), \exists(\neg \exists \alpha) \equiv \neg \exists \alpha, \exists(\exists \alpha+\exists \beta) \equiv \exists \alpha+\exists \beta$, $\exists(\alpha+\alpha)=\exists \alpha+\exists \alpha, \exists(\alpha \cdot \alpha)=\exists \alpha \cdot \exists \alpha$ and closed under modus ponens and necessitation (if $\alpha$, then $\forall \alpha$, where $\forall \alpha=\neg \exists \neg \alpha)$.

Let $L$ denote a first-order language based on $\cdot,+, \rightarrow, \neg, \exists$ and $L_{m}$ denotes monadic propositional language based on $\cdot,+, \rightarrow, \neg, \exists$ and $\operatorname{Form}(L)$ and $\operatorname{Form}\left(L_{m}\right)$ - the set of all formulas of $L$ and $L_{m}$, respectively. We fix a variable $x$ in $L$, associate with each propositional letter $p$ in $L_{m}$ a unique monadic predicate $p^{*}(x)$ in $L$ and define by induction a translation $\Psi: \operatorname{Form}\left(L_{m}\right) \rightarrow \operatorname{Form}(L)$ by putting: $\Psi(p)=p^{*}(x)$ if $p$ is propositional variable, $\Psi(\alpha \circ \beta)=\Psi(\alpha) \circ \Psi(\beta)$, where $\circ=\cdot,+, \rightarrow, \Psi(\exists \alpha)=\exists \Psi(\alpha)$.

Through this translation $\Psi$, we can identify the formulas of $L_{m}$ with monadic formulas of $L$ containing the variable $x$. Moreover, it is routine to check that $\Psi(M L P C) \subseteq Q L$.

\section{Monadic $M V$-algebras}

The characterization of monadic $M V$-algebras as pair of $M V$-algebras, where one of them is a special kind of subalgebra, are given in [3, 2. MV-algebras were introduced by Chang in [1] as an algebraic model for infinitely valued Lukasiewicz logic.

*The author is supported by the grant CNRS-SNRSF N 09/09 and the grant SNRSF N 31/08. 
An $M V$-algebra is an algebra $A=\left(A, \oplus, \odot,{ }^{*}, 0,1\right)$ where $(A, \oplus, 0)$ is an abelian monoid, and the following identities hold for all $x, y \in A: x \oplus 1=1, x^{* *}=x, 0^{*}=1, x \oplus x^{*}=$ $1,\left(x^{*} \oplus y\right)^{*} \oplus y=\left(x \oplus y^{*}\right) \oplus x, x \odot y=\left(x^{*} \oplus y^{*}\right)^{*}$.

An algebra $A=\left(A, \oplus, \odot,{ }^{*}, \exists, 0,1\right)$ is said to be monadic $M V$-algebra (for short $M M V$ algebra) if $A=\left(A, \oplus, \odot,{ }^{*}, 0,1\right)$ is an $M V$-algebra and in addition $\exists$ satisfies the following identities: $x \leq \exists x, \exists(x \vee y)=\exists x \vee \exists y, \exists(\exists x)^{*}=(\exists x)^{*}, \exists(\exists x \oplus \exists y)=\exists x \oplus \exists y, \exists(x \odot y)=\exists x \odot \exists y$, $\exists(x \oplus y)=\exists x \oplus \exists y$.

We shall denote a monadic $M V$-algebra $A=\left(A, \oplus, \odot,{ }^{*}, \exists, 0,1\right)$ by $(A, \exists)$, for brevity. Let $\exists A=\{x \in A: x=\exists x\}$.

A subalgebra $A_{0}$ of an $M V$-algebra $A$ is said to be relatively complete if for every $a \in A$ the set $\left\{b \in A_{0}: a \leq b\right\}$ has the least element.

A subalgebra $A_{0}$ of an $M V$-algebra $A$ is said to be m-relatively complete, if $A_{0}$ is relatively complete and two additional conditions hold:

(\#) $(\forall a \in A)\left(\forall x \in A_{0}\right)\left(\exists v \in A_{0}\right)(x \geq a \odot a \Rightarrow v \geq a \& v \odot v \leq x)$,

(\#\#) $(\forall a \in A)\left(\forall x \in A_{0}\right)\left(\exists v \in A_{0}\right)(x \geq a \oplus a \Rightarrow v \geq a \& v \oplus v \leq x)$.

Proposition 1. 3. Let $\left(A, \oplus, \odot,{ }^{*}, \exists, 0,1\right)$ be a monadic $M V$-algebra. Then the $M V$ subalgebra $\exists A$ of $M V$-algebra $\left(A, \oplus, \odot,{ }^{*}, 0,1\right)$ is m-relatively complete.

Proposition 2. 3. There exists a one-to-one correspondence between.

(1) monadic $M V$-algebras $(A, \exists)$;

(2) the pairs $\left(A, A_{0}\right)$, where $A_{0}$ is $m$-relatively complete subalgebra of $A$.

\section{$3 \quad$ Projective monadic $M V$-algebras}

From the variety of monadic $M V$-algebras $\mathbf{M M V}$ select the subvariety $\mathbf{K}_{\mathbf{n}}$ for $1 \leq n \neq \omega$, which is defined by the following equation [3]: $\left(K_{n}\right) x^{n}=x^{n+1}$, that is $\mathbf{K}_{\mathbf{n}}=\mathbf{M M V}+\left(K_{n}\right)$.

Proposition 3. 3. If $(A, \exists)$ is a totally ordered monadic $M V$-algebra, then $A=\exists A$.

Proposition 4. [3] If $(A, \exists)$ is a finite monadic $M V$-algebra with totally ordered $\exists A$, then $M V$-algebra $A$ is isomorphic to a product of totally ordered $M V$-algebras $A_{i}, i \in I, A_{i} \cong \exists A$ and $\exists A$ is isomorphic to the diagonal subalgebra of the product.

It is defined a unique monadic operator $\exists$ on $S_{n}^{k}$, where $S_{n}=\left(S_{n} ; \oplus, \odot,{ }^{*}, 0,1\right)$ and $S_{n}=\left\{0, \frac{1}{n}, \ldots, \frac{n-1}{n}, 1\right\}$, which corresponds to $m$-relatively complete linearly ordered $M V$ subalgebra, converting the algebra $S_{n}^{k}$ into a simple monadic $M V$-algebra [3. This subalgebra coincides with the greatest diagonal subalgebra, i.e. $d\left(S_{n}^{k}\right)=\left\{(x, \ldots, x) \in S_{n}^{k}: x \in S_{n}\right\}$. Denote this monadic $M V$-algebra by $\left(S_{n}^{k}, \exists_{d}\right)$. In this case the monadic operator $\exists_{d}$ is defined as follows: $\exists_{d}\left(x_{1}, \ldots x_{k}\right)=\left(x_{j}, \ldots, x_{j}\right)$, where $x_{j}=\max \left(x_{1}, \ldots, x_{k}\right)$. The operator $\forall_{d}$ is defined dually: $\forall_{d}\left(x_{1}, \ldots x_{k}\right)=\left(x_{i}, \ldots, x_{i}\right)$, where $x_{i}=\min \left(x_{1}, \ldots, x_{k}\right)$.

Notice that $\mathbf{K}_{\mathbf{n}}$ is generated by $\left(S_{p}^{k}, \exists_{d}\right), p=1, \ldots, n$ and $k \in \omega$. Moreover, $\mathbf{K}_{\mathbf{n}}$ is locally finite and there exists maximal $k \in \omega$, depending on $n$, such that $\left(S_{n}^{k}, \exists_{d}\right)$ is $m$-generated. The maximal $k$ we denote by $t(n)$. There exists also a positive number $r(k, n)$ depending on $k$ and $n$ such that $\left(S_{n}^{k}, \exists_{d}\right)^{r(k, n)}$ is $m$-generated. So,

Theorem 5 .

$$
\prod_{p=1}^{n} \prod_{k=1}^{t(p)}\left(S_{p}^{k}, \exists_{d}\right)^{t(k, p)}
$$


is a free $m$-generated algebra $F_{\mathbf{K}_{\mathbf{n}}}(m)$ in the variety $\mathbf{K}_{\mathbf{n}}$.

Let us notice, that exact description of one-generated free $M M V$-algebra in the variety $\mathbf{K}_{\mathbf{n}}$ is given in 3 .

Theorem 6. The m-generated $M M V$-algebra $A$ from $\mathbf{K}_{\mathbf{n}}$ is projective iff $A$ is isomorphic to $\left(S_{1}^{1}, \exists_{d}\right) \times A^{\prime}$.

Theorem 7. Any subalgebra of the free m-generated algebra $F_{\mathbf{K}_{\mathbf{n}}}(m)$ is projective.

Let $\mathbf{V}_{\mathbf{n}}$ be the variety generated by $\left\{S_{1}, \ldots, S_{n}\right\}$.

Let us observe that

$$
\prod_{p=1}^{n}\left(S_{p}^{1}, \exists\right)^{t(1, p)}
$$

is an algebra with trivial monadic operator $\exists$ (i. e. $\exists x=x$ ) which is isomorphic as an $M V$ algebra to the $m$-generated free $M V$-algebra $F_{\mathbf{V}_{\mathbf{n}}}(m)$. Denote this algebra as $\left(F_{\mathbf{V}_{\mathbf{n}}}(m), \exists\right)$. It holds

Theorem 8. The $M M V$-algebra $\left(F_{\mathbf{V}_{\mathbf{n}}}(m), \exists\right)$ is a retract of the algebra of the free $m$ generated algebra $F_{\mathbf{K}_{\mathbf{n}}}(m)$. So, $\left(F_{\mathbf{V}_{\mathbf{n}}}(m), \exists\right)$ is projective.

\section{Monadic operators on finite $M V$-algebras}

Suppose that $A$ is a finite $M V$-algebra. Then $A \cong S_{n_{1}} \times S_{n_{2}} \times \ldots S_{n_{k}}$ where the $n_{i} \geq 1$. Let $\Pi=\left\{K_{1}, K_{2}, \ldots, K_{m}\right\}$ be a partition of $\{1,2, \ldots, k\}$. We shall say that $\Pi$ is homogeneous if $i, j \in K_{l}$ implies $S_{n_{i}}=S_{n_{j}}$. Given such a $\Pi$, each $K_{i}$ has associated a unique $S_{n_{j}}$ which we shall denote by $A_{i}$. We clearly have $A \cong A_{1}^{K_{1}} \times \ldots \times A_{m}^{K_{m}}$. Since each $K_{i}$ is finite, there is a monadic operator $\exists_{i}$ defined on $A_{i}^{K_{i}}$ such that $\left(A_{i}^{K_{i}}, \exists_{i}\right)$ is an $M M V$-algebra with $\exists_{i}\left(A_{i}^{K_{i}}\right)=A_{i}$. Setting $\exists=\exists_{1} \times \ldots \times \exists_{m}$ and acting pointwise, we obtain a monadic operator $\exists$ on $A$, that is, $(A, \exists)$ is an $M M V$-algebra. If a $K_{i} \in \Pi$ has at least two members, then determined the monadic operator will not be trivial, that is will not be the identity operator.

Proposition 9.2] Suppose that $A$ is a finite $M V$-algebra, say $A=S_{n_{1}} \times S_{n_{2}} \times \ldots S_{n_{k}}$.

(i) For each homogeneous partition $\left\{K_{1}, K_{2}, \ldots, K_{m}\right\}$ of $\{1,2, \ldots, k\}$, there is a monadic operator defined on $A$. Conversely, each monadic operator defined on $A$ is determined by some homogeneous partition of $\left\{K_{1}, K_{2}, \ldots, K_{m}\right\}$.

(ii) If $A=S_{n}^{k}$, then any partition on $\{1,2, \ldots, k\}$ determines a monadic operator on $A$ and conversely, each monadic operator on $A$ comes from some partition of $\{1,2, \ldots, k\}$.

\section{$5 \quad$ Unification problem}

Let $\mathbf{V}$ be a variety of algebras and $F_{\mathbf{V}}(m) m$-generated free algebra over the variety $\mathbf{V}$. Recall that an algebra $A$ of $\mathbf{V}$ is finitely presented if it is a quotient of the form $A=F_{\mathbf{V}}(m) / \theta$, with $\theta$ a finitely generated congruence. Following [4, by an algebraic unification problem we mean a finitely presented algebra $A$ of $\mathbf{V}$. An algebraic unifier for $A$ is a homomorphism $u: A \rightarrow P$ with $P$ a $m$-generated projective algebra in $\mathbf{V}$ and $A$ is algebraically unifiable if such an algebraic unifier exists. Given another algebraic unifier $w: A \rightarrow Q$, we say that $u$ is more general than $w$, written $w \preceq u$, if there is a homomorphism $g: P \rightarrow Q$ such that $w=g u$. The algebraic unification type of an algebraically unifiable finitely presented algebra $A$ in the variety $\mathbf{V}$ is 
now defined exactly as in the symbolic case, using the partially order $\leq$ induced by the quasiorder $\preceq$. Let $U_{\mathbf{V}}(P)$ be the set of unifiers $\sigma: F_{\mathbf{V}}(m) \rightarrow F_{\mathbf{V}}(m)$ for the unification problem $P\left(x_{1}, \ldots, x_{m}\right)$; it is a quasi-ordered set. The problem $P\left(x_{1}, \ldots, x_{m}\right)$ is solvable iff $U_{\mathbf{V}}(P) \neq \emptyset$. Let $(\Sigma, \leq)$ be a poset, where $\leq$ is the ordering induced by the quasi-ordering identifying the equivalence classes with its elements. $\operatorname{Max} \Sigma$ is said to be basis of unifiers for $P$.

We say that an equational theory $E$ has:

1. Unification type 1 iff for every solvable unification problem $P, \operatorname{Card}(\operatorname{Max} \Sigma)=1$.

2. Unification type $\omega$ iff for every solvable unification problem $P, \operatorname{Card}(\operatorname{Max} \Sigma)=n \neq 1$, $n \in \omega$.

3. Unification type $\infty$ iff for every solvable unification problem $P, \operatorname{Card}(\operatorname{Max} \Sigma)$ is infinite.

4. Unification type nullary, if none of the preceding cases applies.

We say that $\mathbf{V}$ has finitary unification type iff it has type 1 or $\omega$.

Theorem 10. The unification type of the equational class $\mathbf{K}_{\mathbf{n}}$ is 1 , i. e. unitary.

\section{References}

[1] C. C. Chang, Algebraic Analysis of Many-Valued Logics, Trans. Amer. Math. Soc., 88(1958), 467490.

[2] L.P. Belluce, R. Grigolia and A. Lettieri, Representations of monadic MV-algebras, Studia Logica, vol. 81, Issue October 15th, 2005, pp. 125-144.

[3] A. Di Nola, R. Grigolia, On Monadic MV-algebras, APAL, Vol. 128, Issues 1-3 (August 2004), pp. 125-139.

[4] S. Ghilardi, Unification through projectivity, J. Logic Comput., 7(6),733-752, 1997.

[5] J.D. Rutledge, A preliminary investigation of the infinitely many-valued predicate calculus, Ph.D. Thesis, Cornell University, 1959. 